\title{
Propiedades mecánicas bajo compresión y resistencia al desgaste abrasivo de piezas gruesas de caucho acrilonitrilo
}

\author{
Thick section acrulonitrile rubber parts mechanical properties \\ under compression and abrasive wear resistance
}

Propriedades mecânicas sob compressão e resistência ao
desgaste abrasivo de peças grossas de borracha de acrilonitrilo

Fecha Recepción: 3 de Diciembre de 2014

Sandra Milena Velásquez-Restrepo*

Diego Hernán Giraldo-Vásquez**

Fecha Aprobación: 29 de Mayo de 2015

Natalia Trinidad Zapata-Gallego***

\section{Resumen}

Se estudió la variación de las propiedades mecánicas bajo compresión uniaxial y el desgaste abrasivo en la sección transversal de piezas gruesas de caucho acrilonitrilo (NBR) reforzadas con negro de humo, teniendo en consideración que en este tipo de piezas las propiedades obtenidas durante la vulcanización no son homogéneas, debido a la baja difusividad térmica de los cauchos. Se realizaron ensayos de desgaste abrasivo en seco, buscando identificar la relación entre la resistencia al desgaste abrasivo y las propiedades mecánicas en compresión, como el módulo elástico y la dureza. Se evaluaron muestras en estado de entrega tras el proceso de vulcanización, luego expuestas al ambiente durante tres meses y, posteriormente, envejecidas en una cámara UV. El envejecimiento ambiental rigidiza los materiales estudiados, pero disminuye la resistencia al desgaste del material proveniente del núcleo de las piezas. El envejecimiento UV disminuyó el módulo elástico, pero su efecto sobre la resistencia al desgaste depende del estado de envejecimiento previo. La dureza no presentó una relación directa ni con el módulo elástico ni con la resistencia al desgaste, por lo cual no puede usarse como propiedad para la selección de cauchos NBR sometidos a desgaste abrasivo. La información obtenida permite generar criterios para el diseño de componentes basados en este tipo de cauchos.

Palabras clave: Caucho NBR, Pruebas de compresión uniaxial, Desgaste abrasivo.

\footnotetext{
* M.Sc. Centro de Diseño y Manufactura del Cuero del SENA (Itagüí-Antioquia, Colombia).smvelasquez@sena.edu.co.

** M.Sc. Universidad de Antioquia (Medellín-Antioquia, Colombia).dhernan.giraldo@udea.edu.co.

*** Universidad de Antioquia (Medellín-Antioquia, Colombia).
} 


\section{Abstract}

In this work the mechanical properties variability under uniaxial compression and the abrasive wear through the cross section of thick-piece carbon-black reinforced acrylonitrile rubber (NBR) parts, was studied. It has to take into account that in these type of pieces, the vulcanized rubber properties heterogeneity causes a variability effect, due to its low thermal diffusivity. Abrasive wear tests under dry conditions were carried out, seeking to identify the relationship between abrasive wear resistance and mechanical properties under uniaxial compression, such as hardness and elastic modulus. The samples in delivery state were evaluated after the vulcanization process, then environmentally exposed during three months and finally UV aged.

Environmental aging stiffens the NBR, but the samples wear resistance from the cross sectional area center, is diminished. Their elastic modulus decreased with the UV aging, but its effect on the wear resistance depends from the previous environmental aging. Their hardness did not show a relationship neither with the elastic modulus nor with the wear resistance; therefore, it should not be used as a property to select the NBR rubbers, subject to wear resistance. The information obtained allows to generate design criteria for nitrile rubber-based parts.

Keywords: NBR rubber, Uniaxial compression tests, Abrasive wear.

\section{Resumo}

Estudou-se a variação das propriedades mecânicas sob compressão uniaxial e o desgaste abrasivo na secção transversal de peças grossas de borracha de acrilonitrilo (NBR) reforçadas com negro de carvão, tendo em consideração que neste tipo de peças as propriedades obtidas durante a vulcanização não são homogêneas, devido à baixa difusividade térmica das borrachas. Realizaram-se ensaios de desgaste abrasivo em seco, buscando identificar a relação entre a resistência ao desgaste abrasivo e as propriedades mecânicas em compressão, como o módulo elástico e a dureza. Avaliaram-se amostras em estado de entrega após o processo de vulcanização, depois de serem expostas ao ambiente durante três meses e, posteriormente, envelhecidas em uma câmara UV. $\mathrm{O}$ envelhecimento ambiental deixa rígidos os materiais estudados, mas diminui a resistência ao desgaste do material proveniente do núcleo das peças. O envelhecimento UV diminuiu o módulo elástico, mas seu efeito sobre a resistência ao desgaste depende do estado de envelhecimento prévio. A dureza não apresentou uma relação direta nem com o módulo elástico nem com a resistência ao desgaste, pelo qual não pode usar-se como propriedade para a seleção de borrachas NBR submetidas ao desgaste abrasivo. A informação obtida permite gerar critérios para o desenho de componentes baseados neste tipo de borrachas.

Palavras chave: Borracha NBR, Provas de compressão uniaxial, Desgaste abrasivo. 


\section{INTRODUCCIÓN}

El caucho nitrilo (NBR, por sus siglas en inglés) es un elastómero copolímero sintético de butadieno y acrilonitrilo, ampliamente usado, gracias a su excelente relación costo/beneficio, en aplicaciones donde se requiere la combinación de propiedades como resistencia a aceites y grasas, bajo valor de compression-set, resistencia al desgaste y resistencia al desgarre [1-4]. El comportamiento de cauchos NBR en condiciones abrasivas ha sido objeto de estudio por su importancia para aplicaciones industriales [3-6], y existe un consenso sobre la dependencia de la resistencia al desgaste abrasivo con la heterogeneidad de la densidad de enlaces entrecruzados [1], la adición de cargas reforzantes nanoestructuradas [2], las propiedades viscoelásticas [3], la temperatura de trabajo [6-7], el sistema de vulcanización [8], la carga normal de contacto [9] y las cargas reforzantes [10-11]; sin embargo, el efecto del envejecimiento sobre la resistencia al desgaste del NBR es aún objeto de estudio.

Molnar et al. reportan que la resistencia al desgaste abrasivo de un NBR que estuvo expuesto al medioambiente durante el servicio en una mina se incrementó con el envejecimiento en condiciones oxidativas [4]. Degrange et al. sometieron a envejecimiento acelerado NBR con diferentes contenidos de acrilonitrilo y reforzados con negro de humo, y encontraron que el material con el mayor contenido de acrilonitrilo mejoró su resistencia al desgaste por deslizamiento con el envejecimiento, mientras que la otra formulación presentó el comportamiento opuesto; los autores afirman que no poder predecir la respuesta frente al desgaste de los NBR cuando se exponen a envejecimiento constituye un serio inconveniente [3].

El esqueleto insaturado del butadieno torna al NBR sensible a la termo-oxidación [12-15], la foto-oxidación [14] y la radiación UV [16-17], factores que junto con el ozono son fuentes de envejecimiento para los cauchos cuando se exponen al medioambiente. En un estudio sobre una banda transportadora fabricada con caucho NBR, la dureza y la resistencia al desgaste abrasivo se incrementaron significativamente con la exposición de la pieza al medioambiente [4]. Se ha reportado que el efecto del envejecimiento térmico en las propiedades de cauchos NBR depende de la formulación empleada. Al someter un NBR reforzado con negro de humo a envejecimiento térmico acelerado, Morrel et al. detectaron un aumento en la densidad de enlaces entrecruzados, lo cual tornó el material más duro y frágil [6], coincidiendo con un trabajo en el que se comparó NBR reforzado con negro de humo y sin reforzar, en el cual ambos materiales se rigidizaron y aumentaron su dureza al exponerlo a termo-oxidación [14]. Degrange et al. obtuvieron un resultado diferente al someter a envejecimiento acelerado NBR reforzado con negro de humo pero con diferentes contenidos de acrilonitrilo, pues no reportan cambios en dureza, módulo elástico o resistencia a la tensión [3]. El efecto del envejecimiento por exposición a radiación UV sobre las propiedades de cauchos NBR también ha sido de interés, y se ha encontrado que aumenta el grado de entrecruzamiento [16-17] y genera escisión de cadenas [17], promoviendo la fragilización y el endurecimiento del material [16-18]. La exposición al ozono presente en el medioambiente favorece el agrietamiento en el NBR [13, 19], debido a la escisión de las cadenas entrecruzadas [20].

Para evaluar la resistencia al desgaste abrasivo de cauchos, el método más representativo [4, 9] es el abrasímetro tipo pin-tambor, de la norma ISO 4649 [21], que emplea un sistema abrasivo a dos cuerpos, sin efecto de los debris en el contacto, dado que durante el recorrido la superficie de la muestra de caucho solo pasa una vez por cada punto del abrasivo, y el efecto cortante de las asperezas de corindón es constante durante el ensayo [22]. Si bien hay estudios sobre desgaste de cauchos que emplean la configuración bola de acero-placa de caucho [1, $3,23]$ o equipos que simulan las condiciones de servicio de piezas industriales [24], no permiten estudiar el desgaste abrasivo de cauchos, pues en esos tribosistemas se combinan diversos tipos de mecanismos de desgaste, como fatiga, adhesión y abrasión, con el inconveniente de la interferencia de los debris, que rápidamente tornan el sistema como abrasión a tres cuerpos, fenómeno que atenúa el mecanismo de desgaste abrasivo, llevando a una sensible disminución en la tasa de desgaste $[1,4]$. La microscopía electrónica de barrido (SEM, por sus siglas en inglés), es la técnica más empleada para identificar los mecanismos de desgaste a partir del 
análisis de las superficies degastadas [1-4, 7, 9-11, 22-24].

Hay estudios que afirman que en los cauchos la resistencia a la abrasión depende de propiedades mecánicas como el módulo elástico y la dureza [2527]. Entre las propiedades mecánicas, el módulo elástico bajo compresión es muy significativo, dado que la mayoría de los productos industriales están sujetos a ese tipo de cargas [10]. La dureza, propiedad ampliamente usada en la industria del caucho para evaluar el estado de los productos vulcanizados [20, 26], se determina mediante un ensayo de indentación bajo compresión, reportándose que existe una marcada relación entre la dureza y el módulo elástico en compresión [25-27].

Los estudios referidos en este documento emplean, generalmente, probetas normalizadas de bajo espesor [1-4, 7-14, 22-24, 26], y solo en algunos casos se evalúan piezas industriales que también son delgadas, como láminas [18] y sellos toroidales [5-6]. Sin embargo, es bien conocido que durante la vulcanización de artículos en caucho no se logra que las propiedades sean homogéneas en la sección transversal; su baja difusividad térmica hace que en piezas de más de $10 \mathrm{~mm}$ de espesor la temperatura varíe significativamente en función de la distancia a la superficie, por lo tanto, el grado de vulcanización también varía a lo largo de la sección transversal [28]. Para tener en cuenta este comportamiento de los cauchos, se han desarrollado estudios a muestras extraídas de artículos industriales, como bandas transportadoras [4], llantas [15, 24] o piezas moldeadas con líneas de partición [18]. Esta metodología permite evaluar el estado de materiales vulcanizados en condiciones reales de proceso, generando información relevante para diseñar, fabricar o estudiar el desempeño de piezas en caucho.

En este trabajo se estudian el módulo elástico en compresión uniaxial, la dureza y la resistencia al desgaste abrasivo en seco de probetas extraídas de la superficie y de la sección transversal de una pieza gruesa de caucho, vulcanizada con un caucho NBR reforzado con negro de humo. Se evalúa el efecto de la exposición al medioambiente, del envejecimiento acelerado bajo radiación UV y del sitio de obtención de las probetas en las propiedades mencionadas, identificando los mecanismos de desgaste abrasivo.

\section{Materiales y Métodos}

En todas las condiciones de ensayo se empleó una formulación constituida por $57.7 \% \mathrm{p} / \mathrm{p}$ de caucho NBR y aditivos de bajo peso molecular, reforzada con $39.6 \% \mathrm{p} / \mathrm{p}$ de negro de humo y $2.7 \% \mathrm{p} / \mathrm{p}$ de cargas minerales. Se estudiaron piezas vulcanizadas en forma anular, con un diámetro externo de 425 $\mathrm{mm}$ y un diámetro interno de $349 \mathrm{~mm}$, de sección rectangular, como se muestra en la Fig. 1. Se evaluaron anillos de $56 \mathrm{~mm}$ y de $78 \mathrm{~mm}$ de ancho, de tal manera que sus secciones transversales correspondían a rectángulos de $38 \mathrm{~mm} \times 56 \mathrm{~mm}$ y de $38 \mathrm{~mm} \times 78 \mathrm{~mm}$, respectivamente.

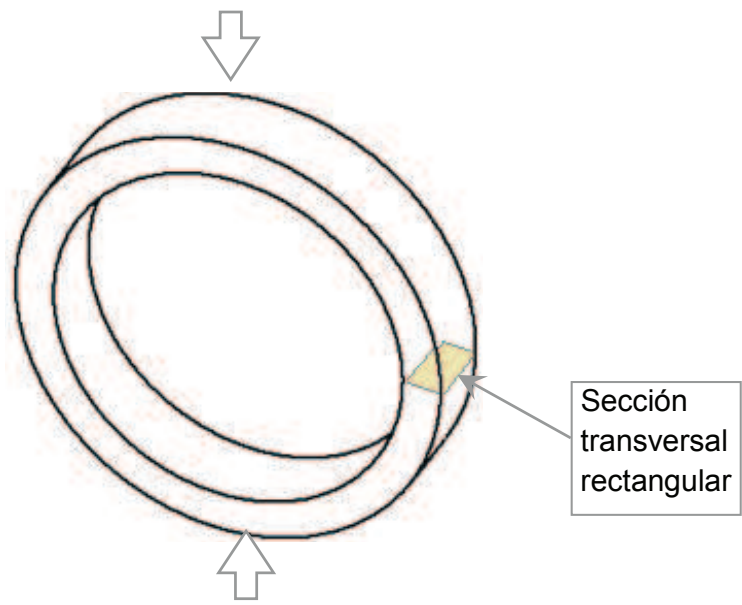

Fig. 1. Esquema ilustrativo sobre el método de aplicación de la carga compresiva uniaxial durante los ensayos no destructivos de rigidez a las piezas completas. Las flechas indican el sentido de aplicación de la carga.

Se realizó un estudio exploratorio sobre la rigidez de ambos tipos de anillos en estado de entrega luego de la vulcanización, y de uno de los anillos de $56 \mathrm{~mm}$ de ancho que fue sometido a envejecimiento ambiental durante tres meses en la parte superior de una torre del nororiente de la ciudad de Medellín (Colombia). Las tres muestras fueron sometidas a ensayos de compresión uniaxial, aplicando la fuerza compresiva en sentido diametral hasta deformarlos $35 \mathrm{~mm}$, como se muestra en la Fig. 1. Se calculó la rigidez a partir de la curva carga aplicada-deformación en compresión. Los ensayos se efectuaron en una máquina universal de ensayos marca Shimadzu, empleando una celda de 
carga de $100 \mathrm{KN}$ con una sensibilidad de $0.5 \mathrm{~N}$ y una velocidad de mordazas de $20 \mathrm{~mm} /$ minuto.

Se analizaron muestras de $56 \mathrm{~mm}$ y $78 \mathrm{~mm}$ de ancho, recordando que como los diámetros interior y exterior se conservaron constantes, la altura de la sección transversal es de $38 \mathrm{~mm}$ en las piezas. En la Tabla 1 se indican las características de las tres piezas a las que se les evaluó la rigidez en compresión mediante ensayos no destructivos.

\section{TABLA 1}

IDENTIFICACIÓN DE LAS MUESTRAS SOMETIDAS A ENSAYOS NO DESTRUCTIVOS DE RIGIDEZ

\begin{tabular}{|l|l|c|}
\hline \multicolumn{1}{|c|}{ Estado del material } & \multicolumn{1}{|c|}{$\begin{array}{c}\text { Dimensiones de la sección } \\
\text { rectangular }\end{array}$} & Código de identificación \\
\hline \multirow{2}{*}{ En estado de entrega de la vulcanización } & $\begin{array}{l}56 \mathrm{~mm} \text { de ancho por } 38 \mathrm{~mm} \mathrm{de} \\
\text { altura }\end{array}$ & Delg-Nue \\
\cline { 2 - 4 } & $\begin{array}{l}78 \mathrm{~mm} \text { de ancho por } 38 \mathrm{~mm} \mathrm{de} \\
\text { altura }\end{array}$ & Anch-Nue \\
\hline $\begin{array}{l}\text { Expuesta al medioambiente durante } 3 \\
\text { meses }\end{array}$ & $\begin{array}{l}56 \mathrm{~mm} \text { de ancho por } 38 \mathrm{~mm} \mathrm{de} \\
\text { altura }\end{array}$ & Delg-Exp \\
\hline
\end{tabular}

Los tres tipos de anillos fueron cortados como se esquematiza en la Fig. 2, con el fin de obtener bloques de los cuales se pudieran extraer cilindros de $25 \mathrm{~mm}$ de diámetro para realizar ensayos de compresión uniaxial, y de $12 \mathrm{~mm}$ de diámetro para ensayos de desgaste abrasivo. Se efectuaron cortes rectos en dirección radial y perimetral con una sierra de corte tipo sinfín, refrigerando con agua a temperatura ambiente, con el fin de evitar el sobrecalentamiento del caucho. Para obtener los cilindros se emplearon sacabocados acoplados a un taladro de banco, refrigerando también con agua. Los cuerpos de prueba cilíndricos se obtuvieron en sentido transversal y perimetral, como se esquematiza en la Fig. 2.
La obtención de probetas de zonas que estuvieron en contacto con las paredes del molde y del núcleo de la pieza permite evaluar si existen diferencias entre las propiedades de ambas regiones de las piezas. Es pertinente llamar la atención sobre el hecho de que una de las caras planas de las probetas en sentido transversal estuvo en contacto con el molde durante la vulcanización, de tal manera que la distancia a las paredes del molde varía en el sentido axial de la probeta. Para las probetas extraídas en sentido perimetral se obtuvieron del centro de la sección transversal, por lo tanto, la distancia a las paredes del molde es la misma a lo largo de toda la longitud del cilindro.

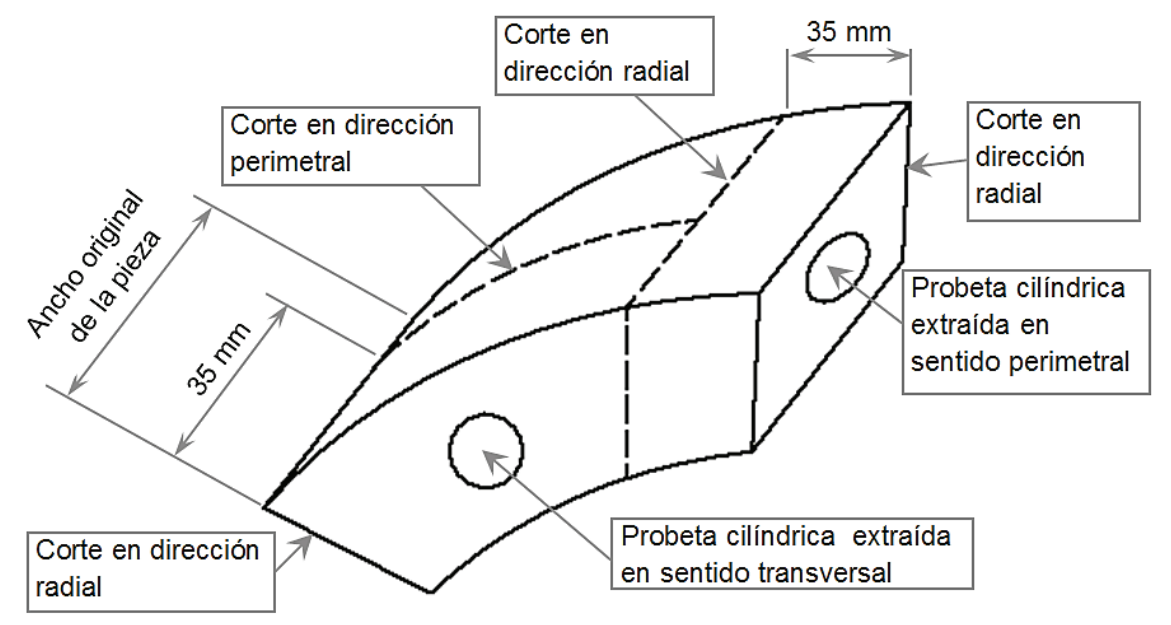

Fig. 2. Esquema que ilustra la manera como se efectuaron los cortes en dirección radial y transversal para obtener las probetas cilíndricas para los ensayos de compresión uniaxial y desgaste abrasivo. 
En la Tabla 2 se resumen las condiciones de los cilindros usados como cuerpos de prueba, y los códigos usados para identificar las muestras.

TABLA 2

IDENTIFICACIÓN DE LOS CILINDROS EVALUADOS

\begin{tabular}{|c|c|c|c|c|}
\hline \multirow{2}{*}{\begin{tabular}{|c|} 
Estado de \\
envejecimiento del \\
material \\
\end{tabular}} & \multirow{2}{*}{$\begin{array}{c}\text { Ancho de } \\
\text { la sección } \\
\text { transversal } \\
\end{array}$} & \multirow{2}{*}{$\begin{array}{c}\text { Origen de los } \\
\text { cuerpos de } \\
\text { prueba } \\
\end{array}$} & \multicolumn{2}{|c|}{ Código de identificación } \\
\hline & & & $\begin{array}{c}\text { Antes de } \\
\text { envejecimiento UV }\end{array}$ & Envejecidas en cámara UV \\
\hline \multirow{2}{*}{$\begin{array}{l}\text { Estado de entrega de } \\
\text { la vulcanización }\end{array}$} & \multirow{2}{*}{$56 \mathrm{~mm}$} & Perimetral & Delg-Vul-P & Delg-Vul-UV-P \\
\hline & & Transversal & Delg-Vul-T & Delg-Vul-UV-T \\
\hline \multirow{2}{*}{$\begin{array}{l}\text { Estado de entrega de } \\
\text { la vulcanización }\end{array}$} & \multirow{2}{*}{$78 \mathrm{~mm}$} & Perimetral & Anch-Vul-P & Anch-Vul-UV-P \\
\hline & & Transversal & Anch-Vul-T & Anch-Vul-UV-T \\
\hline \multirow{2}{*}{$\begin{array}{l}\text { E n v e j e c i d a } \\
\text { ambientalmente }\end{array}$} & \multirow{2}{*}{$56 \mathrm{~mm}$} & Perimetral & Delg-EnvAmb-P & Delg-EnvAmb-UV-P \\
\hline & & Transversal & Delg-EnvAmb-T & Delg-EnvAmb-UV-T \\
\hline
\end{tabular}

Se realizaron los tres experimentos descritos en la Tabla 3, en la cual se indican los factores considerados y las variables respuesta. Para cada experimento y cada variable respuesta se realizó un análisis de varianza (ANOVA, por sus siglas en inglés). En todos los casos, el nivel de significancia fue 0.05 , y se aplicó la prueba de Tukey para identificar diferencias entre medias.

\section{Tabla 3}

\section{DISEÑO EXPERIMENTAL EMPLEADO}

\begin{tabular}{|c|c|c|c|}
\hline $\begin{array}{c}\text { Tipo de } \\
\text { experimento }\end{array}$ & \begin{tabular}{|c|} 
Factores considerados en el \\
experimento
\end{tabular} & Niveles & Variables respuesta \\
\hline \multirow{2}{*}{$\begin{array}{l}\text { Diseño factorial } \\
\text { completo } 2^{2}\end{array}$} & Envejecimiento ambiental & $\begin{array}{l}\text { En estado de entrega de la } \\
\text { vulcanización y envejecida } \\
\text { ambientalmente }\end{array}$ & \multirow{2}{*}{$\begin{array}{l}\text { - Módulo elástico en } \\
\text { compresión } \\
\text { - Dureza }\end{array}$} \\
\hline & $\begin{array}{l}\text { Sitio de la pieza del que se } \\
\text { extraen los cuerpos de prueba }\end{array}$ & Perimetral y transversal & \\
\hline \multirow{3}{*}{$\begin{array}{l}\text { Diseño factorial } \\
\text { completo } 2^{3}\end{array}$} & $\begin{array}{|lll|}\begin{array}{l}\text { Espesor de la pieza } \\
\text { vulcanizada }\end{array} & \\
\end{array}$ & $56 \mathrm{mmy} 78 \mathrm{~mm}$ & \multirow{3}{*}{$\begin{array}{l}\text { - Módulo elástico en } \\
\text { compresión } \\
\text { - Dureza }\end{array}$} \\
\hline & Envejecimiento UV & Sin y con envejecimiento UV & \\
\hline & $\begin{array}{l}\text { Sitio de la pieza del que se } \\
\text { extraen los cuerpos de prueba }\end{array}$ & Perimetral y transversal & \\
\hline \multirow{3}{*}{$\begin{array}{l}\text { Diseño factorial } \\
\text { completo } 2^{3}\end{array}$} & Envejecimiento ambiental & $\begin{array}{l}\text { En estado de entrega de la } \\
\text { vulcanización y envejecida } \\
\text { ambientalmente }\end{array}$ & \multirow{3}{*}{$\begin{array}{l}\text { - Pérdida de volumen } \\
\text { por desgaste abrasivo }\end{array}$} \\
\hline & Envejecimiento UV & Sin y con envejecimiento UV & \\
\hline & $\begin{array}{l}\text { Sitio de la pieza del que se } \\
\text { extraen los cuerpos de prueba }\end{array}$ & Perimetral y transversal & \\
\hline
\end{tabular}

Los ensayos de compresión uniaxial se realizaron bajo norma ASTM D 695, en una máquina universal de ensayos marca Shimadzu, evaluando tres muestras para cada condición de ensayo. Se empleó una celda de carga de $100 \mathrm{KN}$ con una sensibilidad de $0.5 \mathrm{~N}$ y una velocidad de mordazas de $5 \mathrm{~mm} / \mathrm{min}$. 
Tres cilindros de cada condición de ensayo fueron sometidos a envejecimiento UV bajo la norma ASTM G-154 en una cámara marca UV Q-Lab, durante 36 horas; luego de extraerlas de la cámara UV se aclimataron a temperatura ambiente durante 24 horas, determinando su dureza Shore A en las caras planas de las probetas bajo la norma ASTM D2240, empleando un durómetro marca CEAST, y realizando ensayos de compresión uniaxial.

La pérdida de volumen por desgaste abrasivo se determinó en un abrasímetro Maqtest, siguiendo las recomendaciones de la norma ISO 4649, utilizando un papel abrasivo marca SATRA con partículas abrasivas de corindón. La cara en contacto con el molde de las probetas extraídas en sentido transversal fue la que se puso en contacto con el abrasivo. Para las probetas extraídas del núcleo de la pieza se eligieron aleatoriamente las caras que se pusieron en contacto con el abrasivo. A cada muestra se le calculó la pérdida de volumen a partir de la pérdida de masa, empleando una balanza analítica marca BA 210S para calcular la densidad de las muestras. Las superficies desgastadas se analizaron por microscopía electrónica de barrido en un equipo JEOL JSM-6490LV.

\section{Resultados y Discusión}

\section{A. Pruebas de rigidez a las piezas completas}

La Tabla 4 presenta los valores de rigidez obtenidos para los anillos en sentido diametral, al realizar los ensayos de compresión no destructivos.

TABLA 4

RIGIDEZ DE LOS ANILLOS EN SENTIDO DIAMETRAL

\begin{tabular}{|c|l|c|}
\hline $\begin{array}{c}\text { Espesor de la pieza } \\
(\mathbf{m m})\end{array}$ & \multicolumn{1}{|c|}{ Estado de la pieza } & Rigidez (N/mm) \\
\hline 56 & Estado de entrega de la vulcanización & 6.61 \\
\hline 56 & Envejecida ambientalmente & 10.22 \\
\hline 78 & Estado de entrega de la vulcanización & 13.02 \\
\hline
\end{tabular}

Como era esperado, la rigidez de la muestra más ancha es superior a la del anillo con menor espesor, pues a mayor área de soporte de carga aumenta la rigidez. El envejecimiento ambiental aumentó la rigidez, como ha sido reportado en algunos estudios para cauchos NBR reforzados con negro de humo [6, 14, 16-18].

\section{B. Efecto del envejecimiento ambiental y del sitio de la pieza del que se extraen los cuerpos de prueba en el módulo elástico y la dureza}

En la Fig. 3 se presentan los diagramas de cajas para el módulo elástico bajo compresión uniaxial y para la dureza. 

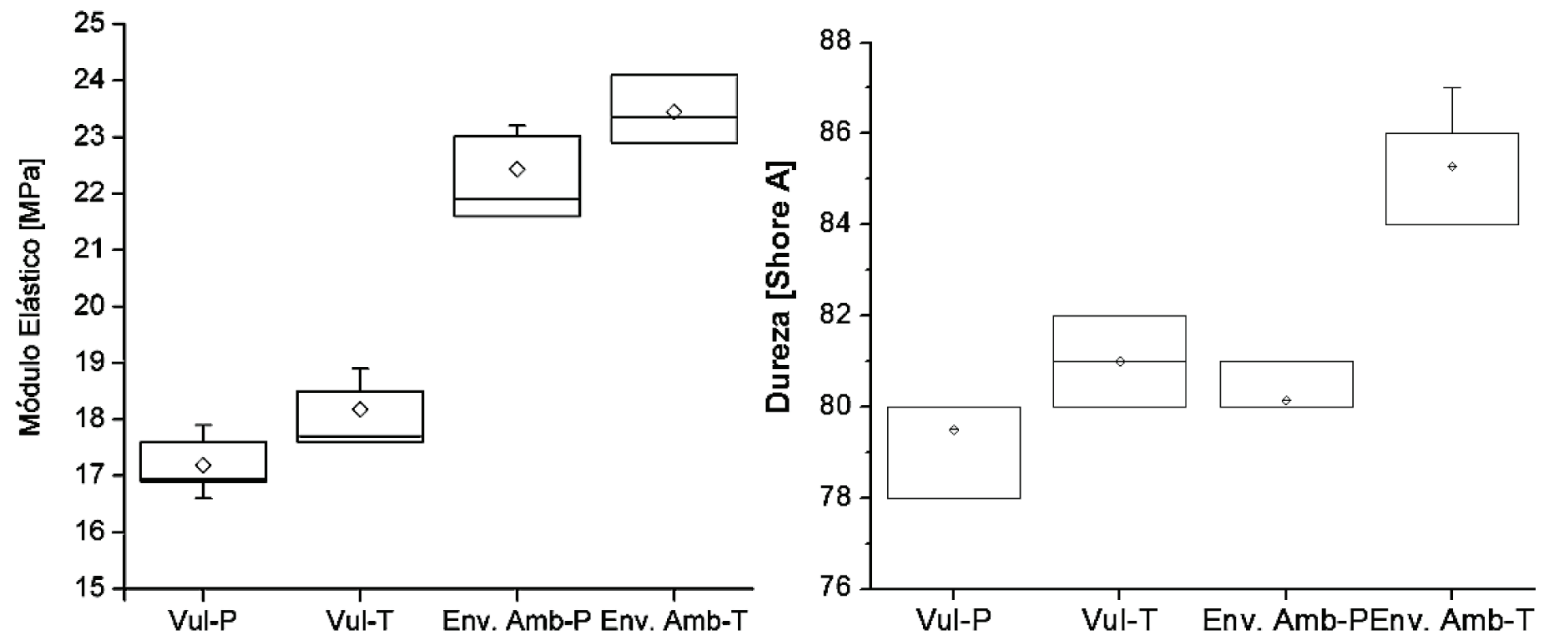

Fig. 3. Efecto del sitio de extracción de los cuerpos de prueba y del envejecimiento ambiental en el módulo elástico en compresión uniaxial y en la dureza.

El análisis ANOVA permitió establecer que tanto el envejecimiento ambiental como el sitio de la pieza del que provienen las probetas incide en el módulo elástico y en la dureza del NBR estudiado. El envejecimiento ambiental aumentó el módulo elástico en compresión uniaxial, resultado que concuerda con lo observado en los ensayos de compresión no destructivos a las piezas completas. Como se ha señalado previamente en este trabajo, en varios estudios se ha reportado el aumento en la rigidez y la dureza de cauchos NBR reforzados con negro de humo como efecto del envejecimiento ambiental, debido al aumento en la densidad de enlaces entrecruzados como efecto de la termo-oxidación $[6,14,16-18]$. Las muestras provenientes de la zona en contacto con el molde durante la vulcanización presentan valores de módulo elástico y de dureza superiores a las muestras del núcleo de la pieza; este comportamiento se debe a la heterogeneidad en la vulcanización en piezas gruesas, la cual, como fue explicado en una sección anterior de este documento, se presenta en piezas con espesores superiores a $10 \mathrm{~mm}$ como resultado de la baja difusividad térmica de los cauchos.

\section{Efecto del envejecimiento UV, del espesor de la pieza y del sitio de la pieza del que se extraen los cuerpos de prueba en el módulo elástico y la dureza}

En la Fig. 4 se presentan los diagramas de cajas para el módulo elástico bajo compresión uniaxial, y en la Fig. 5, para la dureza. El análisis ANOVA permitió establecer que el envejecimiento UV y el sitio de la pieza del que provienen las probetas inciden en el módulo elástico y en la dureza del NBR estudiado. También permitió identificar que el espesor de la pieza también incide en el módulo elástico, pero no presentó un efecto estadísticamente significativo sobre la dureza. 


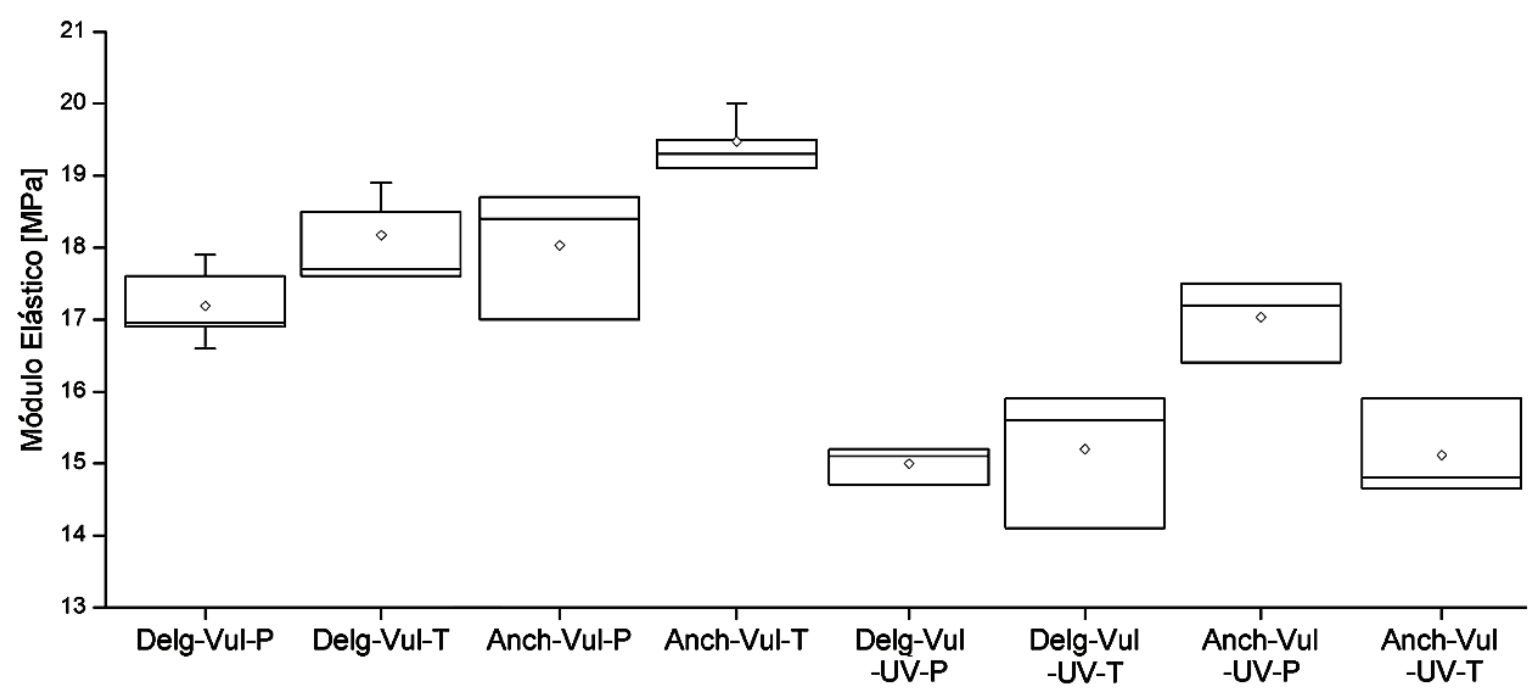

Fig. 4. Efecto del envejecimiento UV, del espesor de la pieza y del sitio de extracción de los cuerpos de prueba en el módulo elástico en compresión uniaxial.

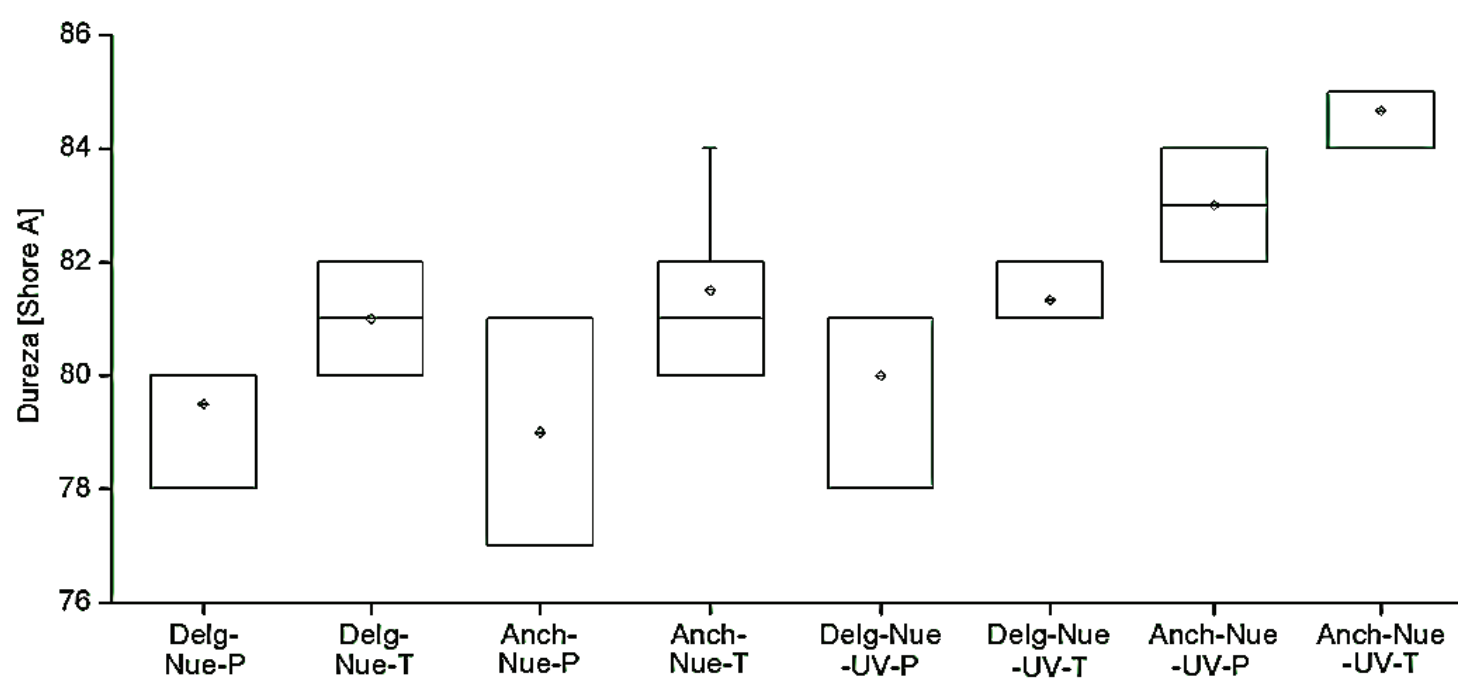

Fig. 5. Efecto del envejecimiento UV, del espesor de la pieza y del sitio de extracción de los cuerpos de prueba en el módulo elástico y la dureza.

La radiación UV produce escisión de cadenas, disminuyendo la rigidez del material, pero es una degradación de la zona superficial de las muestras, como ha sido reportado en otros estudios [16-18]. La diferencia encontrada en la respuesta del módulo elástico y la dureza como efecto de la radiación UV se debe a que mientras que el módulo elástico es una propiedad volumétrica, la dureza Shore A determina el estado de un pequeño volumen de la zona aledaña a la región deformada por acción del indentador, siendo entonces una medición mucho más sensible a pequeñas variaciones que existan en el material como resultado del mezclado de los ingredientes del caucho o de la vulcanización.

\section{Efecto del envejecimiento ambiental, del} envejecimiento $\mathrm{U} V$ y del sitio de la pieza del que se extraen los cuerpos de prueba en el desgaste

\section{abrasivo}

En la Fig. 6 se presentan los diagramas de cajas para la pérdida de volumen durante las pruebas de desgaste abrasivo. El análisis ANOVA permitió establecer 
que ni el envejecimiento UV, ni el envejecimiento ambiental, ni el sitio de la pieza del que se extraen los cuerpos de prueba, ni las interacciones entre esos factores tienen incidencia en la pérdida de volumen por desgaste abrasivo. Solo se apreció una disminución significativa de la resistencia al desgaste para las probetas extraídas del núcleo y que fueron sometidas a envejecimiento ambiental, independientemente de si fueron expuestas a envejecimiento UV o no, lo cual confirma que la exposición al ozono logra conservar la resistencia al desgaste abrasivo de las muestras de la zona superficial de la pieza. Esta independencia de la resistencia al desgaste abrasivo con el envejecimiento ambiental o el envejecimiento UV no ha sido reportada previamente, pues en estudios desarrollados sobre piezas industriales se había encontrado un incremento en la resistencia al desgaste como efecto del envejecimiento ambiental [4] o del envejecimiento térmico para una formulación de caucho NBR [3], o una disminución, para una formulación de caucho NBR diferente a esta última [3].

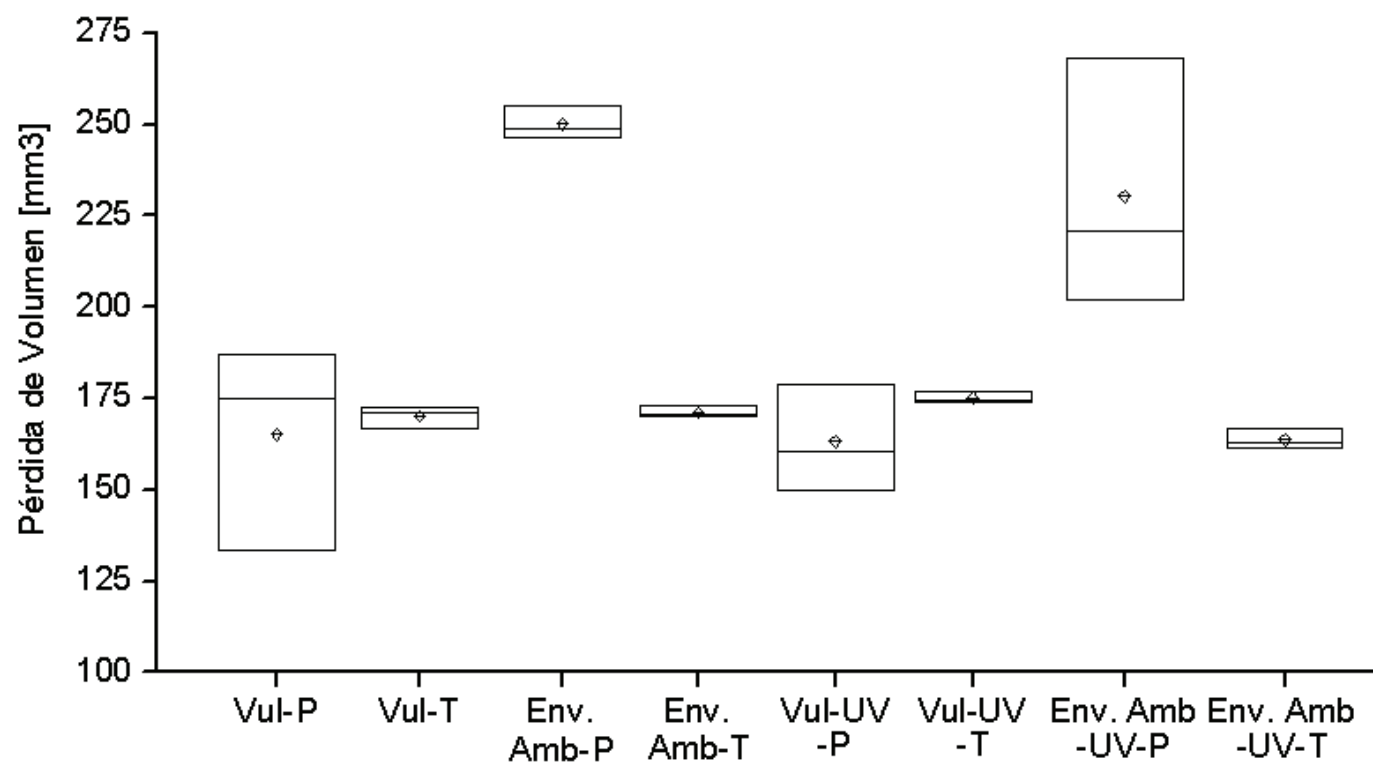

Fig. 6. Efecto del envejecimiento ambiental, del envejecimiento UV y del sitio de extracción de los cuerpos de prueba en la pérdida de volumen por abrasión.

En la Fig. 7 se presenta la pérdida de volumen durante los ensayos de desgaste abrasivo, en función del módulo elástico. 


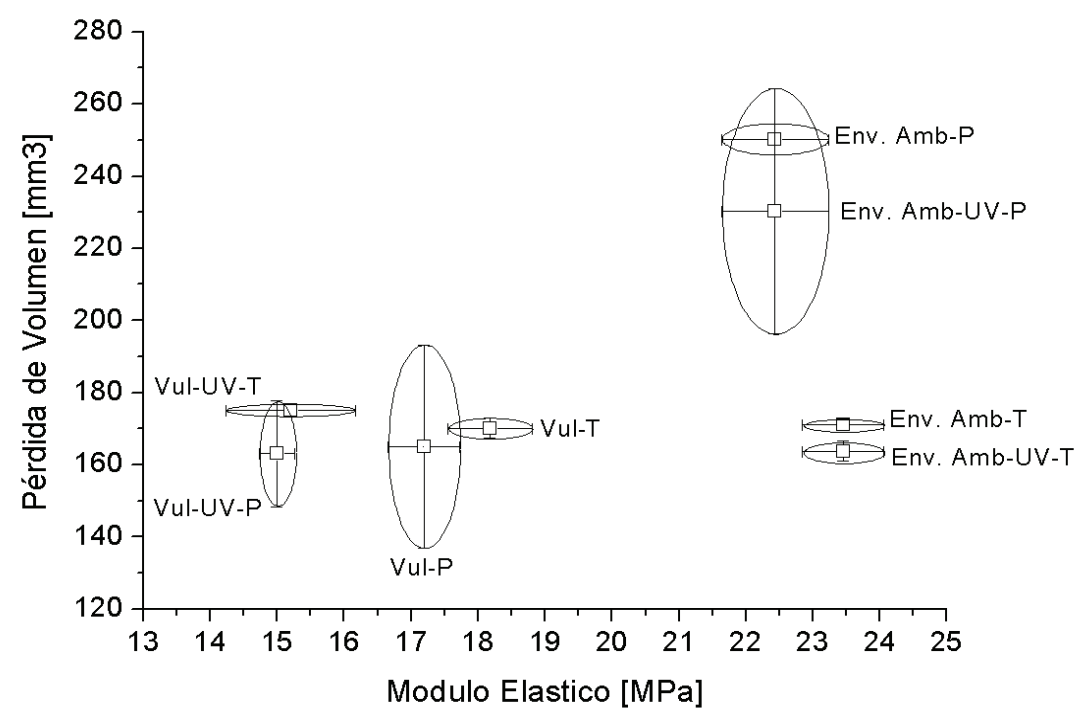

Fig. 7. Pérdida de volumen durante los ensayos de desgaste abrasivo versus el módulo elástico. Se presenta la desviación estándar del módulo elástico y de la pérdida de volumen por desgaste abrasivo.

En la Fig. 8 se muestran imágenes obtenidas por microscopía electrónica de barrido de las superficies desgastadas.

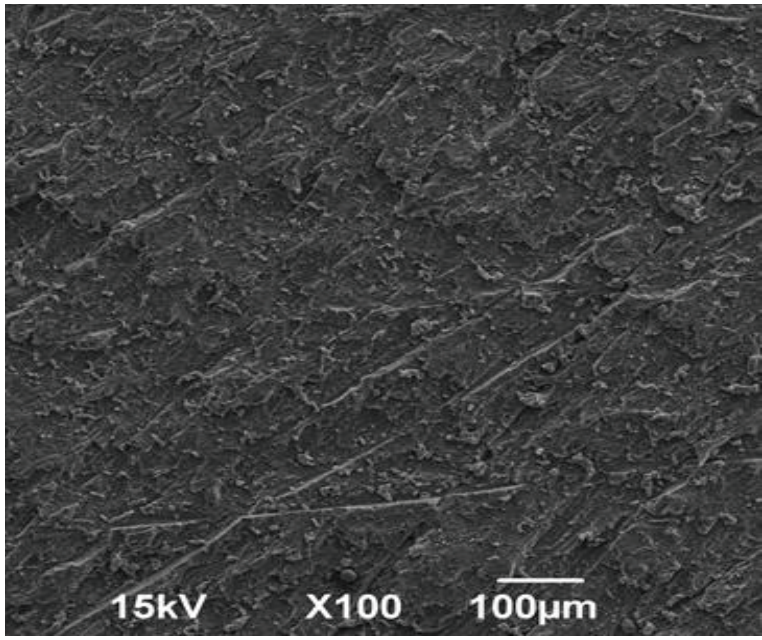

(a) Estado de entrega de la vulcanización- Transversal

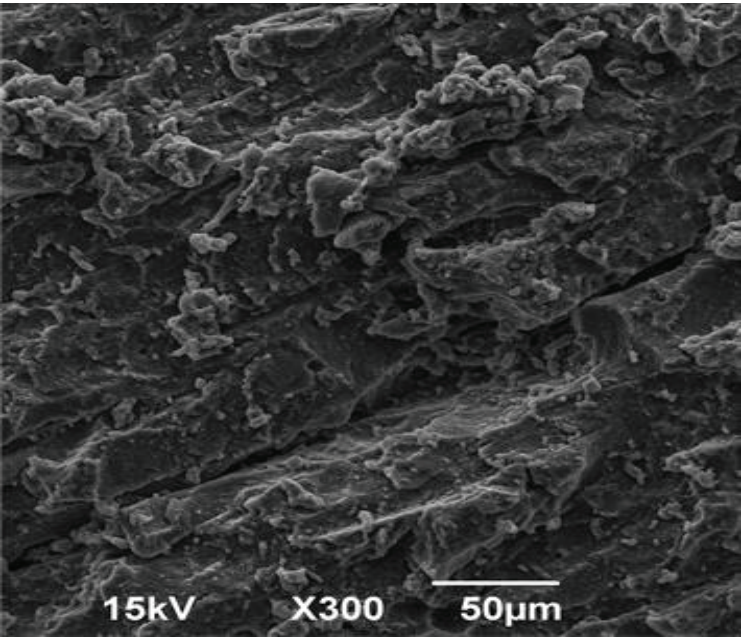

(b) Estado de entrega de la vulcanización Envejecimiento UV-Transversal 


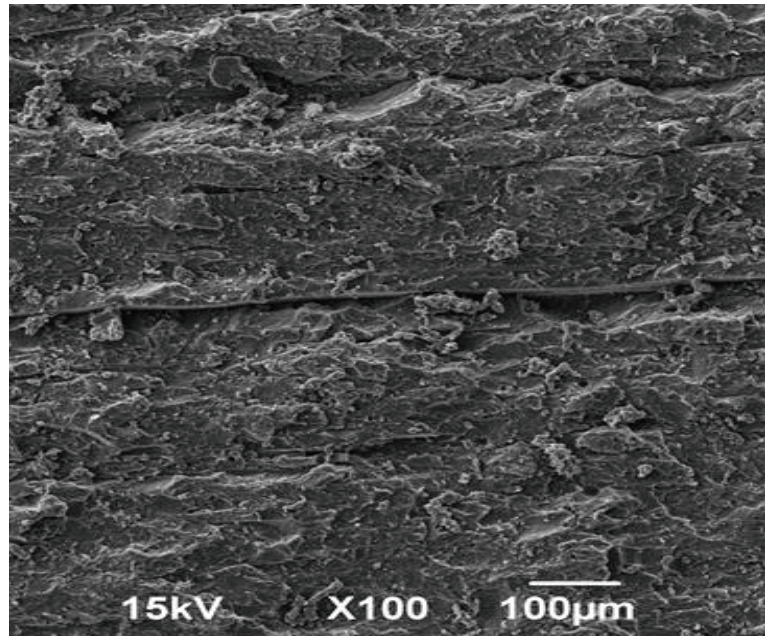

(c) Envejecimiento ambiental - Perimetral

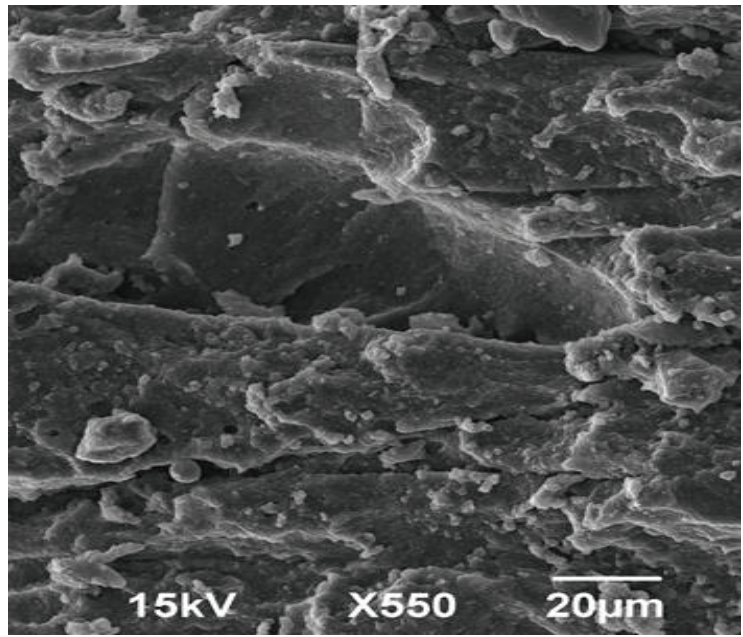

(d) Envejecimiento ambientalmente y UV- Perimetral

FiG. 8. Imágenes obtenidas por microscopía electrónica de barrido de las superficies desgastadas.

No se apreciaron diferencias significativas entre las morfologías de las superficies desgastadas para las diferentes condiciones de ensayo. En todas las superficies se aprecian surcos en la dirección del movimiento de las partículas abrasivas, indicando la acción cortante de algunas partículas abrasivas del corindón en los ensayos bajo norma ISO 4649 [23, 11], así como ondas de Schallamach típicas del desgaste abrasivo de los cauchos en abrasión $[1,6]$. No se encontraron rollos formados a partir de la acción de los debris, como se espera cuando se estudia desgaste abrasivo en los que no se pasa repetidamente por la misma superficie y se atenúa el efecto abrasivo de las partículas del contra cuerpo [4, 22], ni cráteres asociados a fatiga [29].

Mientras que durante el ensayo de dureza Shore A y de compresión uniaxial los esfuerzos generan deformación viscoelástica [20], los surcos y las ondas observados en las superficies desgastadas corresponden a una deformación viscoplástica generada por el desplazamiento de las partículas abrasivas durante el contacto, lo cual explica que no exista una relación entre la resistencia al desgaste abrasivo y propiedades mecánicas como la dureza Shore A y el módulo elástico, como sí ocurre con otros tipos de materiales.

\section{Conclusiones}

La resistencia al desgaste abrasivo de los cauchos NBR, en las condiciones de ensayo empleadas en este trabajo, no presenta una relación directa con la dureza ni con el módulo elástico, comportamiento que debe ser considerado por los responsables del diseño o la selección de materiales.

Es común el uso de cauchos NBR en situaciones en las que se exponen al medio ambiente, en donde la temperatura, la radiación UV y el ozono alteran las propiedades del material por diversos mecanismos de degradación; pero no existe aún un consenso sobre el efecto de algunas condiciones de envejecimiento en propiedades tan relevantes como el módulo elástico, la dureza Shore A y la resistencia al desgaste abrasivo. La heterogeneidad en la sección transversal de las propiedades de piezas gruesas en caucho, entonces, debe ser considerada cuando se realicen estudios sistemáticos sobre degradación, propiedades mecánicas o resistencia al desgaste de este tipo de materiales.

En este trabajo, el envejecimiento ambiental rigidizó el material estudiado, tanto sobre muestras de la superficie de las piezas moldeadas como del material proveniente del núcleo, pero el envejecimiento UV solo afectó las propiedades del material extraído 
de la superficie. La resistencia al desgaste abrasivo del material de la superficie de las piezas no fue afectada por el envejecimiento ambiental ni por el envejecimiento UV, como es deseado si se piensa en la durabilidad de los productos, pero el material del núcleo sí fue afectado.

Si bien los cauchos NBR son materiales ampliamente utilizados en diversos tipos de industrias, es pertinente generar información sobre propiedades mecánicas y desgaste, aplicando metodologías que permitan evaluar el desempeño en condiciones lo más cercanas posibles a las de servicio.

\section{Agradecimientos}

A la Universidad de Antioquia, por la financiación del proyecto MDC 10-1-01, que permitió realizar este trabajo.

\section{REFERENCIAS}

[1] Z. Cao, D. Wang, C. Cong, Y. Wang and Q. Zhou, "Dependence of abrasion behavior on cross-linked heterogeneity in unfilled nitrile rubber", Tribology International, vol. 69, pp. 141-149, 2014.

[2] B. Likozar and Z. Major, "Morphology, mechanical, cross-linking, thermal, and tribological properties of nitrile and hydrogenated nitrile rubber/multi-walled carbon nanotubes composites prepared by melt compounding: The effect of acrylonitrile content and hydrogenation", Applied Surface Science, vol. 257, Issue 2,pp. 565-573, Nov. 2010.

[3] J. Degrange, M. Thomine, P. Kapsa, J. Pelletier, L. Chazeau, G. Vigier, G. Dudragne and L. Guerbé, "Influence of viscoelasticity on the tribological behaviour of carbon black filled nitrile rubber (NBR) for lip seal application", Wear, vol. 259, no. 1-6, pp. 684-692, Jul. 2005.

[4] W. Molnar, M. Varga, P. Braun, K. Adam and E. Badisch, "Correlation of rubber based conveyor belt properties and abrasive wear rates under 2and 3-body conditions", Wear, Volume 320, pp. 1-6, December 2014.

[5] J. Yamabe and S. Nishimura, "Influence of fillers on hydrogen penetration properties and blister fracture of rubber composites for O-ring exposed to high-pressure hydrogen gas", International Journal on Hydrogen Energy, no. 34, pp. 1977-1989, 2009.

[6] P. Morrell, M. Patel and A.Skinner, "Accelerated thermal ageing studies on nitrile rubber O-rings", Polymer Testing, no. 22,pp. 651-656, 2003.

[7] D. Higgins, B. Marmo, C. Jeffree, V. Koutsos and J. Blackford, "Morphology of ice wear from rubber-ice friction tests and its dependence on temperature and sliding velocity", Wear, no. 265,pp. 634-644,2008.

[8] K. El-Nemr, "Effect of different curing systems on the mechanical and physico-chemical properties of acrylonitrile butadiene rubber vulcanizates", Materials \& Design, vol. 32, no. 6, pp. 3361-3369, Jun. 2011

[9] K. Pal, S. Pal, C. Das and J. Kim, "Relationship between normal load and dynamic coefficient of friction on rock-rubber wear mechanism", Materials \& Design, vol. 31, no. 10, pp. 47924799, Dec. 2010.

[10] A. Mostafa, A. Abouel-Kasem, M. Bayoumi, and M. El-Sebaie, "Insight into the effect of CB loading on tension, compression, hardness and abrasion properties of SBR and NBR filled compounds", Materials\& Design, vol. 30, no. 5, pp. 1785-1791, May 2009.

[11] K. Pal, T. Das, R. Rajasekar, S. Pal, Chapal K. Das, "Wear characteristics of styrene butadiene rubber/natural rubber blends with varying carbon blacks by DIN abrader and mining rock surfaces", Journal of Applied Polymer Science, vol. 111, pp. 348-357,2009.

[12] M. Garbarczyk, W. Kuhn, J. Klinowski and S. Jurga, "Characterization of aged nitrile rubber elastomers by NMR Spectroscopy and microimaging", Polymer, vol.43, pp. 31693172, 2002.

[13] P. Budrugeac, E. Segal and S. Ciutacu, "Termooxidative degradation of nitrile butadiene rubber", Journal of Thermal Analysis, vol. 37, pp. 1179-1191,1991.

[14] F. Delor-Jestin, N. Barrois-Oudin, C. Cardinet, J. Lacoste and J. Lemaire, "Thermal ageing of acrylonitrile-butadiene copolymer", Polymer Degradation and Stability, vol. 70, no. 1, pp. 1-4, 2000. 
[15] J. Baldwin, D. Bauer and K. Ellwood, "Accelerated aging of tires, Part II", Rubber Chemistry and Technology, vol. 78, pp. 336353, May 2005.

[16] H.Katoh, R. Kamotoand J.Murata, "Degradation analysis of NBR and epichlorohydrin rubber by new micro analysis method", e-Journal of Soft Materials, vol. 2,pp. 20-24, 2006.

[17] R. Yang, J. Zhao and Y. Liu, "Oxidative degradation products analysis of polymer materials by pyrolysis gas chromatographymass spectrometry", Polymer Degradation and Stability, vol. 98, no. 12, pp. 2466-2472,Dec. 2013.

[18] S. Patcharaphun, W. Chookaew and T. Tungkeunkunt, "Influence of thermal and oil aging on weldline strength of NR/EPDM and NR/NBR blends", Kasetsart Journal: Natural Science, vol. 45, no. 5, pp. 909-916, 2011.

[19] D. Bielinski, D. Pieczynska, U. Ostaszewska and J. Jagielski, "Modification of rubber by ion bombardment", Nuclear Instruments and Methods in Physics Research Section B: Beam Interactions with Materials and Atoms, vol. 282, no. 1,pp. 141-144, July 2012.

[20] A. Gent and W. Mars, "Strength of Elastomers", The Science and Technology of Rubber, New York, NY, USA: Academic Press, pp. 473-516, 2013.

[21] ISO, "ISO 4649: Rubber,vulcanizedor thermoplastic - determination of abrasion resistance using arotating cylindrical drumdevice", 2002.

[22] D. Allsopp, R. Trezona, I. Hutchings, "The effects of ball surface condition in the micro- scale abrasive weartest", Tribology Letter,vol. 5, pp 259-64,1998.

[23] Y. Guo, J. Wang, K. Li and X. Ding, "Tribological properties and morphology of bimodal elastomeric nitrilebutadiene rubber networks", Materials \& Design, vol.52, pp. 861-869, 2013.

[24] E. Euchler, O. Kratina, R. StoIek, M. Gehde, "A study of correlation between crack initiation during dynamic wear process and fatigue crack growth of reinforced rubber materials", European Symposium on Friction, Wear, and Wear Protection, Karlsruhe, Germany, Conference Papers in Science,2015.

[25] B. Wang, J. Hua, and Y. Ping, "Application of artificial neural network in prediction of abrasion of rubber composites", Mater. Des., vol. 49, pp. 802-807, 2013.

[26] S. Rios, R. Chicurel, and L. Del Castillo, "Potential of particle and fibre reinforcement of tyre tread elastomer", Materials \& Design, no. 22, pp. 369-374, 2001.

[27] N. Viswanath, D. Bellow, "Development of an equation for the wear of polymers", Wear, vol. 181-183,part I, pp. 42-49, 1995.

[28] S. Nozu, H. Tsuji and K. Onishi, "A prediction model for rubber curing process", Heat Transfer - Engineering Applications, Croatia: Vyacheslav Vikhrenko (Ed.), In Tech, pp. 151-170, 2011.

[29] G. Petitet, J. Loubet, H. Zahouani and D. Mazuyer, "A contribution to the understanding of elementary wear mechanisms of rubber filled compounds", Rubber Chemistry and Technology, vol. 78, pp. 312-320,2005. 